\title{
A RELATIONSHIP BETWEEN CHARACTERISTIC VALUES AND VECTORS
}

\author{
E. T. BEASLEY, JR. AND P. M. GIBSON
}

\begin{abstract}
It is shown that for all nonzero $n$-component column vectors $\alpha$ and $\beta$ over a field $F$ there exists a set $\Gamma$ of $n$-square matrices over $F$ of cardinality $n^{2}-2 n+2$ such that, for each $n$-square matrix $A$ over $F, A \alpha=\alpha$ or $A^{T} \beta=\beta$ if and only if 1 is a characteristic value of $P A$ for every $P \in \Gamma$.
\end{abstract}

Let $F$ be a field with unit 1 , and $A$ an $n$-square matrix over $F$. If all row sums of $A$ or all row sums of $A^{T}$ (the transpose of $A$ ) are 1 , then $A$ is a stochastic matrix. It is obvious that if $A$ is stochastic then 1 is a characteristic value of $P A$ for every $n$-square permutation matrix $P$. R. A. Brualdi and $\mathrm{H}$. W. Wielandt [1] proved the converse. In this paper, it is shown as a corollary to a more general result that the $n$ ! permutation matrices can be replaced by a set of $n$-square matrices of cardinality $n^{2}-2 n+2$.

Let $F^{n}$ be the set of all $n \times 1$ column vectors over $F$, and let $\alpha, \beta \in F^{n}-$ $\{0\}$. A set $\Gamma$ of $n$-square matrices over $F$ is called an $(\alpha, \beta)$-set provided that, for each $n$-square matrix $A$ over $F, A \alpha=\alpha$ or $A^{T} \beta=\beta$ if and only if 1 is a characteristic value of $P A$ for every $P \in \Gamma$. An $(\alpha, \beta)$-set $\Gamma$ is minimal if no proper subset of $\Gamma$ is an $(\alpha, \beta)$-set. If $\alpha^{T} \beta=0$, then $\alpha$ and $\beta$ are orthogonal. Let $\varepsilon_{k}$ be the vector in $F^{n}$ with all components zero, except component $k$, which is equal to 1 . Denote the vector in $F^{n}$ with all components equal to 1 by $\varepsilon$. The characterization of stochastic matrices by Brualdi and Wielandt implies that the set of all $n$-square permutation matrices is an $(\varepsilon, \varepsilon)$-set of cardinality $n$ !. We shall show that minimal $(\alpha, \beta)$-sets of cardinality $n^{2}-2 n+2$ can be easily constructed for all $\alpha, \beta \in F^{n}-\{0\}$.

We first exhibit minimal $(\alpha, \beta)$-sets for two particular choices of $\alpha$ and $\beta$. One of these is $\alpha=\beta=\varepsilon_{1}$, and the other is $\alpha=\varepsilon_{1}$ and $\beta=\varepsilon_{n}$.

Lemma 1. For each positive integer $n$, the set

$$
\Phi_{n}=\left\{\varepsilon_{1} \varepsilon_{1}^{T}\right\} \cup\left\{\varepsilon_{1} \varepsilon_{1}^{T}+\varepsilon_{i} \varepsilon_{j}^{T} \mid i, j=2,3, \cdots, n\right\}
$$

of $n$-square matrices over $F$ is a minimal $\left(\varepsilon_{1}, \varepsilon_{1}\right)$-set.

Presented to the Society November 17, 1973; received by the editors March 28, 1973.

AMS (MOS) subject classifications (1970). Primary 15A18; Secondary 15A51.

(c) American Mathematical Society 1974 
Proof. Let $A=\left(a_{i j}\right)$ be an $n$-square matrix over $F$. Since $\varepsilon_{1}^{T} \varepsilon_{1}=1$ and $\varepsilon_{j}^{T} \varepsilon_{1}=0$ for $j=2,3, \cdots, n$,

$$
\begin{array}{rlrl}
A \varepsilon_{1}=\varepsilon_{1} & \Rightarrow P A \varepsilon_{1}=\varepsilon_{1} & & \forall P \in \Phi_{n}, \\
A^{T} \varepsilon_{1}=\varepsilon_{1} & \Rightarrow(P A)^{T} \varepsilon_{1}=\varepsilon_{1} & \forall P \in \Phi_{n} .
\end{array}
$$

Hence, if $A \varepsilon_{1}=\varepsilon_{1}$ or $A^{T} \varepsilon_{1}=\varepsilon_{1}$, then 1 is a characteristic value of $P A$ for every $P \in \Phi_{n}$. Now suppose that 1 is a characteristic value of $P A$ for every $P \in \Phi_{n}$. Since 1 is a characteristic value of $\varepsilon_{1} \varepsilon_{1}^{T} A, a_{11}=1$. Since 1 is a characteristic value of $\left(\varepsilon_{1} \varepsilon_{1}^{T}+\varepsilon_{j} \varepsilon_{i}^{T}\right) A$,

$$
\operatorname{det}\left(\left(\varepsilon_{1} \varepsilon_{1}^{T}+\varepsilon_{j} \varepsilon_{i}^{T}\right) A-I\right)=0, \quad i, j=2,3, \cdots, n .
$$

This implies that

$$
\operatorname{det}\left[\begin{array}{cc}
a_{11}-1 & a_{1 j} \\
a_{i 1} & a_{i j}-1
\end{array}\right]=0, \quad i, j=2,3, \cdots, n .
$$

Therefore, since $a_{11}=1, a_{i 1} a_{1 j}=0, i, j=2,3, \cdots, n$. Since $a_{11}=1$, this implies that $A \varepsilon_{1}=\varepsilon_{1}$ or $A^{T} \varepsilon_{1}=\varepsilon_{1}$. Hence, $\Phi_{n}$ is an $\left(\varepsilon_{1}, \varepsilon_{1}\right)$-set. We now show that $\Phi_{n}$ is minimal. If

$$
A=\varepsilon \varepsilon_{1}^{T}+\varepsilon_{1} \varepsilon^{T}-2 \varepsilon_{1} \varepsilon_{1}^{T},
$$

then $A \varepsilon_{1}=A^{T} \varepsilon_{1} \neq \varepsilon_{1}$, and equation (1) holds. This implies that $\Phi_{n}-$ $\left\{\varepsilon_{1} \varepsilon_{1}^{T}\right\}$ is not an $\left(\varepsilon_{1}, \varepsilon_{1}\right)$-set. If $k, m \in\{2,3, \cdots, n\}$ and

$$
A=\varepsilon_{1} \varepsilon_{1}^{T}+\varepsilon_{1} \varepsilon_{k}^{T}+\varepsilon_{m} \varepsilon_{1}^{T},
$$

then $A \varepsilon_{1} \neq \varepsilon_{1} \neq A^{T} \varepsilon_{1}, a_{11}=1$, and equation (1) holds except for $i=m$ and $j=k$. This implies that

$$
\Phi_{n}-\left\{\varepsilon_{1} \varepsilon_{1}^{T}+\varepsilon_{k} \varepsilon_{m}^{T}\right\}, \quad k, m=2,3, \cdots, n,
$$

is not an $\left(\varepsilon_{1}, \varepsilon_{1}\right)$-set. Therefore, $\Phi_{n}$ is a minimal $\left(\varepsilon_{1}, \varepsilon_{1}\right)$-set.

Lemma 2. Let $E_{1 n}=\varepsilon_{1} \varepsilon_{1}^{T}+\varepsilon_{n} \varepsilon_{n}^{T}$. For each integer $n>1$, the set

$$
\begin{aligned}
\Psi_{n}= & \left\{E_{1 n}, E_{1 n}+\varepsilon_{1} \varepsilon_{n}^{T}\right\} \\
& \cup\left\{E_{1 n}+\varepsilon_{1} \varepsilon_{j}^{T}, E_{1 n}+\varepsilon_{i} \varepsilon_{n}^{T}, E_{1 n}+\varepsilon_{1} \varepsilon_{j}^{T}+\varepsilon_{i} \varepsilon_{n}^{T} \mid i, j=2,3, \cdots, n-1\right\}
\end{aligned}
$$

of $n$-square matrices over $F$ is a minimal $\left(\varepsilon_{1}, \varepsilon_{n}\right)$-set.

Proof. Let $A=\left(a_{i j}\right)$ be an $n$-square matrix over $F$. Then

$$
\begin{array}{rlrl}
A \varepsilon_{1} & =\varepsilon_{1} \Rightarrow E_{1 n} A \varepsilon_{1}=\varepsilon_{1}, \varepsilon_{i} \varepsilon_{j+1}^{T} A \varepsilon_{1}=0, & & i, j=1,2, \cdots, n-1, \\
A^{T} \varepsilon_{n} & =\varepsilon_{n} \Rightarrow\left(E_{1} A\right)^{T} \varepsilon_{n}=\varepsilon_{n},\left(\varepsilon_{i} \varepsilon_{j+1}^{T} A\right)^{T} \varepsilon_{n}=0, & i, j=1,2, \cdots, n-1 .
\end{array}
$$


Hence, if $A \varepsilon_{1}=\varepsilon_{1}$ or $A^{T} \varepsilon_{n}=\varepsilon_{n}$, then 1 is a characteristic value of $P A$ for every $P \in \Psi_{n}$. Suppose that 1 is a characteristic value of $P A$ for every $P \in \Psi_{n}$. Since 1 is a characteristic value of $E_{1 n} A$ and $\left(E_{1 n}+\varepsilon_{1} \varepsilon_{n}^{T}\right) A$,

$$
\begin{gathered}
\operatorname{det}\left[\begin{array}{cc}
a_{11}-1 & a_{1 n} \\
a_{n 1} & a_{n n}-1
\end{array}\right]=0, \\
\operatorname{det}\left[\begin{array}{cc}
a_{11}+a_{n 1}-1 & a_{1 n}+a_{n n} \\
a_{n 1} & a_{n n}-1
\end{array}\right]=0 .
\end{gathered}
$$

Equations (2) and (3) imply that $a_{n 1}=0$. Then equation (2) implies that $a_{11}=1$ or $a_{n n}=1$. Hence, if $n=2$, then $A \varepsilon_{1}=\varepsilon_{1}$ or $A^{T} \varepsilon_{n}=\varepsilon_{n}$. Suppose that $n>2$. We complete the proof that $\Phi_{n}$ is an $\left(\varepsilon_{1}, \varepsilon_{n}\right)$-set by considering three cases.

CASE 1. $a_{11}=1, a_{n n} \neq 1$. Since 1 is a characteristic value of $\left(E_{1 n}+\varepsilon_{1} \varepsilon_{i}^{T}\right) A$,

(4) $\operatorname{det}\left[\begin{array}{cc}a_{11}+a_{i 1}-1 & a_{1 n}+a_{i n} \\ a_{n 1} & a_{n n}-1\end{array}\right]=0, \quad i=2,3, \cdots, n-1$.

Since $a_{n 1}=0, a_{11}=1$, and $a_{n n} \neq 1$, equation (4) implies that $a_{i 1}=0$ for $i=2,3, \cdots, n-1$. Therefore, $A \varepsilon_{1}=\varepsilon_{1}$.

CASE 2. $a_{11} \neq 1, a_{n n}=1$. Since 1 is a characteristic value of $\left(E_{1 n}+\varepsilon_{j} \varepsilon_{n}^{T}\right) A$,

(5) $\operatorname{det}\left[\begin{array}{ccc}a_{11}-1 & a_{1 j} & a_{1 n} \\ a_{n 1} & a_{n j}-1 & a_{n n} \\ a_{n 1} & a_{n j} & a_{n n}-1\end{array}\right]=0, \quad j=2,3, \cdots, n-1$.

Since $a_{n 1}=0, a_{11} \neq 1$, and $a_{n n}=1$, equation (5) implies that $a_{n j}=0$ for $j=2,3, \cdots, n-1$. Hence, $A^{T} \varepsilon_{n}=\varepsilon_{n}$.

CASE 3. $a_{11}=1=a_{n n}$. Since 1 is a characteristic value of

(6)

$$
\operatorname{det}\left[\begin{array}{ccc}
\left(E_{1 n}+\varepsilon_{1} \varepsilon_{i}^{T}+\varepsilon_{j} \varepsilon_{n}^{T}\right) A \\
a_{11}+a_{i 1}-1 & a_{1 j}+a_{i j} & a_{1 n}+a_{i n} \\
a_{n 1} & a_{n j}-1 & a_{n n} \\
a_{n 1} & a_{n j} & a_{n n}-1
\end{array}\right]=0
$$

for $i, j=2,3, \cdots, n-1$. Therefore, since $a_{n 1}=0$ and $a_{11}=1=a_{n n}$,

$$
a_{i 1} a_{n j}=0, \quad i, j=2,3, \cdots, n-1,
$$

and we see that $A \varepsilon_{1}=\varepsilon_{1}$ or $A^{T} \varepsilon_{n}=\varepsilon_{n}$. 
We now show that $\Psi_{n}$ is minimal. If

$$
A=\sum_{k=2}^{n} \varepsilon_{k} \varepsilon_{1}^{T}+\sum_{m=2}^{n-1} \varepsilon_{1} \varepsilon_{m}^{T}-\sum_{k, m=2}^{n-1} \varepsilon_{k} \varepsilon_{m}^{T}
$$

then it is not difficult to show that $A \varepsilon_{1} \neq \varepsilon_{1}$ and $A^{T} \varepsilon_{n} \neq \varepsilon_{n}$, while equations (3) through (6) hold. This implies that $\Psi_{n}-\left\{E_{1 n}\right\}$ is not an $\left(\varepsilon_{1}, \varepsilon_{n}\right)$-set. If $A=\varepsilon_{1} \varepsilon_{n}^{T}+\varepsilon_{n} \varepsilon_{1}^{T}$, then $A \varepsilon_{1} \neq \varepsilon_{1}$ and $A^{T} \varepsilon_{n} \neq \varepsilon_{n}$, while equations (2), (4), (5), and (6) hold. This implies that $\Psi_{n}-\left\{E_{1 n}+\varepsilon_{1} \varepsilon_{n}^{T}\right\}$ is not an $\left(\varepsilon_{1}, \varepsilon_{n}\right)$-set. If $m \in\{2,3, \cdots, n-1\}$ and $A=\varepsilon_{1} \varepsilon_{1}^{T}+\varepsilon_{m} \varepsilon_{1}^{T}$, then $A \varepsilon_{1} \neq \varepsilon_{1}$ and $A^{T} \varepsilon_{n} \neq \varepsilon_{n}$, while equations (2) through (6) hold except when $i=m$ in equation (4). This implies that

$$
\Psi_{n}-\left\{E_{1 n}+\varepsilon_{1} \varepsilon_{m}^{T}\right\}, \quad m=2,3, \cdots, n-1,
$$

is not an $\left(\varepsilon_{1}, \varepsilon_{n}\right)$-set. If $k \in\{2,3, \cdots, n-1\}$ and $A=\varepsilon_{n} \varepsilon_{k}^{T}+\varepsilon_{n} \varepsilon_{n}^{T}$, then $A \varepsilon_{1} \neq \varepsilon_{1}$ and $A^{T} \varepsilon_{n} \neq \varepsilon_{n}$, while equations (2) through (6) hold except when $j=k$ in equation (5). This implies that

$$
\Psi_{n}-\left\{E_{1 n}+\varepsilon_{k} \varepsilon_{n}^{T}\right\}, \quad k=2,3, \cdots, n-1,
$$

is not an $\left(\varepsilon_{1}, \varepsilon_{n}\right)$-set. If $k, m \in\{2,3, \cdots, n-1\}$ and $A=E_{1 n}+\varepsilon_{m} \varepsilon_{1}^{T}+\varepsilon_{n} \varepsilon_{k}^{T}$, then $A \varepsilon_{1} \neq \varepsilon_{1}$ and $A^{T} \varepsilon_{n} \neq \varepsilon_{n}$, while equations (2) through (6) hold except when $i=m$ and $j=k$ in equation (6). This implies that

$$
\Psi_{n}-\left\{E_{1 n}+\varepsilon_{1} \varepsilon_{m}^{T}+\varepsilon_{k} \varepsilon_{n}^{T}\right\}, \quad k, m=2,3, \cdots, n-1,
$$

is not an $\left(\varepsilon_{1}, \varepsilon_{n}\right)$-set. Hence, $\Psi_{n}$ is a minimal $\left(\varepsilon_{1}, \varepsilon_{n}\right)$-set.

Lemma 3. Let $\alpha, \beta, \gamma, \delta \in F^{n}-\{0\}$, and let $P$ and $Q$ be nonsingular matrices over $F$ such that

$$
P \gamma=\alpha, \quad P^{T} \beta=c \delta, \quad Q \alpha=\gamma, \quad Q^{T} c \delta=\beta,
$$

where $c \in F-\{0\}$. If $\Gamma$ and $\Gamma^{\prime}$ are sets of n-square matrices over $F$ such that

$$
\Gamma^{\prime}=\{P B Q \mid B \in \Gamma\},
$$

then $\Gamma^{\prime}$ is an $(\alpha, \beta)$-set if and only if $\Gamma$ is a $(\gamma, \delta)$-set.

Proof. Suppose that $\Gamma$ is a $(\gamma, \delta)$-set, and let $A$ be an $n$-square matrix over $F$. Assume that

$$
A \alpha=\alpha \text { or } A^{T} \beta=\beta .
$$

Since (7) holds, this implies that $A P \gamma=Q^{-1} \delta$ or $A^{T} Q^{T} \delta=\left(P^{T}\right)^{-1} \delta$. Therefore,

$$
(Q A P) \gamma=\gamma \text { or }(Q A P)^{T} \delta=\delta .
$$


Hence, since $\Gamma$ is a $(\gamma, \delta)$-set, 1 is a characteristic value of $B(Q A P)$ for every $B \in \Gamma$. If $G=P B Q$, then $B(Q A P)$ and $G A$ are similar matrices. Therefore, from (8) we see that 1 is a characteristic value of $G A$ for every $G \in \Gamma^{\prime}$. Reversing this sequence of steps, we see that $A \alpha=\alpha$ or $A^{T} \beta=\beta$ if 1 is a characteristic value of $G A$ for every $G \in \Gamma^{\prime}$. Therefore, $\Gamma^{\prime}$ is an $(\alpha, \beta)$-set. A similar argument shows that $\Gamma$ is a $(\gamma, \delta)$-set if $\Gamma^{\prime}$ is an $(\alpha, \beta)$-set.

It is convenient to express our principal results on minimal $(\alpha, \beta)$-sets in two theorems. One of these applies when $\alpha^{T} \beta \neq 0$, and the other when $\alpha^{T} \beta=0$.

THEOREM 1. Let $\alpha, \beta \in F^{n}$ with $\alpha^{T} \beta \neq 0$. If $\left\{\gamma_{i}, \delta_{i} \mid i=1,2, \cdots, n-1\right\}$ is a subset of $F^{n}$ such that $\left\{\alpha, \gamma_{1}, \gamma_{2}, \cdots, \gamma_{n-1}\right\}$ and $\left\{\beta, \delta_{1}, \delta_{2}, \cdots, \delta_{n-1}\right\}$ are independent while $\alpha$ and $\beta$ are orthogonal to $\delta_{i}$ and $\gamma_{i}$, respectively, for $i=1,2, \cdots, n-1$, then

$$
\Gamma=\left\{\left(\alpha^{T} \beta\right)^{-1} \alpha \beta^{T}\right\} \cup\left\{\left(\alpha^{T} \beta\right)^{-1} \alpha \beta^{T}+\gamma_{i} \delta_{j}^{T} \mid i, j=1,2, \cdots, n-1\right\}
$$

is a minimal $(\alpha, \beta)$-set.

Proof. Let $P$ be the $n$-square matrix with columns $\alpha, \gamma_{1}, \gamma_{2}, \cdots$, $\gamma_{n-1}$, and let $Q$ be the $n$-square matrix with columns $\beta^{\prime}, \delta_{1}, \delta_{2}, \cdots, \delta_{n-1}$, where $\beta^{\prime}=\left(\alpha^{T} \beta\right)^{-1} \beta$. Then $P$ and $Q$ are nonsingular with

$$
P \varepsilon_{1}=\alpha, \quad P^{T} \beta=\left(\alpha^{T} \beta\right) \varepsilon_{1}, \quad Q^{T} \alpha=\varepsilon_{1}, \quad Q\left(\alpha^{T} \beta\right) \varepsilon_{1}=\beta .
$$

Since $\Gamma=\left\{P B Q^{T} \mid B \in \Phi_{n}\right\}$, it follows from Lemmas 1 and 3 that $\Gamma$ is a minimal $(\alpha, \beta)$-set.

THEOREM 2. Let $\alpha, \beta \in F^{n}-\{0\}$ with $\alpha^{T} \beta=0$. Suppose that

$$
\left\{\gamma_{i}, \delta_{i} \mid i=1,2, \cdots, n-1\right\}
$$

is a subset of $F^{n}$ such that $\left\{\alpha, \gamma_{1}, \gamma_{2}, \cdots, \gamma_{n-1}\right\}$ and $\left\{\beta, \delta_{1}, \delta_{2}, \cdots, \delta_{n-1}\right\}$ are independent, $\alpha^{T} \delta_{1}=1=\beta^{T} \gamma_{n-1}$, and $\alpha$ and $\beta$ are orthogonal to $\delta_{i+1}$ and $\gamma_{i}$, respectively, for $i=1,2, \cdots, n-2$. If $H=\alpha \delta_{1}^{T}+\gamma_{n-1} \beta^{T}$, then

$$
\begin{aligned}
\Gamma= & \left\{H, H+\alpha \beta^{T}\right\} \\
& \cup\left\{H+\alpha \delta_{j+1}^{T}, H+\gamma_{i} \beta^{T}, H+\alpha \delta_{j+1}^{T}+\gamma_{i} \beta^{T} \mid i, j=1,2, \cdots, n-2\right\}
\end{aligned}
$$

is a minimal $(\alpha, \beta)$-set.

Proof. Let $P$ be the $n$-square matrix with columns $\alpha, \gamma_{1}, \gamma_{2}, \cdots$, $\gamma_{n-1}$, and let $Q$ be the $n$-square matrix with columns $\delta_{1}, \delta_{2}, \cdots, \delta_{n-1}, \beta$. Then $P$ and $Q$ are nonsingular with

$$
P \varepsilon_{1}=\alpha, \quad P^{T} \beta=\varepsilon_{n}, \quad Q^{T} \alpha=\varepsilon_{1}, \quad Q \varepsilon_{n}=\beta .
$$


Since $\Gamma=\left\{P B Q^{T} \mid B \in \Psi_{n}\right\}$, it follows from Lemmas 2 and 3 that $\Gamma$ is a minimal $(\alpha, \beta)$-set.

Let $\alpha, \beta \in F^{n}-\{0\}$. Clearly a subset $\left\{\gamma_{i}, \delta_{i} \mid i=1,2, \cdots, n-1\right\}$ of $F^{n}$ exists satisfying the conditions of Theorems 1 or 2 according to whether $\alpha^{T} \beta \neq 0$ or $\alpha^{T} \beta=0$. Hence we have the following.

COROLlaRY 1. If $\alpha, \beta \in F^{n}-\{0\}$, there exists a minimal $(\alpha, \beta)$-set of cardinality $n^{2}-2 n+2$.

If we let $\alpha=\beta=\varepsilon$ in this corollary we obtain the following.

COROLlaRY 2. There exists a set $\Gamma$ of $n$-square matrices over $F$ of cardinality $n^{2}-2 n+2$ such that, for each $n$-square matrix $A$ over $F, A$ is stochastic if and only if 1 is a characteristic value of $P A$ for every $P \in \Gamma$.

Consideration of $(\alpha, \beta)$-sets was motivated by Brualdi and Wielandt's remark [1] on the difficulty of finding sets of fewer than $n$ ! permutation matrices which could be used in their characterization of the $n$-square stochastic matrices. We have no general results on this problem, but we can show that every set of five 3-square permutation matrices is a minimal $(\varepsilon, \varepsilon)$-set.

Let $\Gamma$ be the set of all 3-square permutation matrices over $F$, excluding the identity matrix. Let $A=\left(a_{i j}\right)$ be a 3 -square matrix over $F$. Suppose that 1 is a characteristic value of $P A$ for every $P \in \Gamma$. Then

$$
\operatorname{det}(I-P A)=0 \quad \forall P \in \Gamma .
$$

If we multiply each matrix $I-P A$ on the left by $P^{T}$, we obtain

$$
\operatorname{det}(P-A)=0 \quad \forall P \in \Gamma .
$$

Let

$$
\Delta=\left\{\left[\begin{array}{ll}
0 & 1 \\
1 & 0
\end{array}\right],\left[\begin{array}{ll}
0 & 1 \\
0 & 0
\end{array}\right],\left[\begin{array}{ll}
0 & 0 \\
1 & 0
\end{array}\right],\left[\begin{array}{ll}
1 & 0 \\
0 & 0
\end{array}\right],\left[\begin{array}{ll}
0 & 0 \\
0 & 1
\end{array}\right]\right\} .
$$

If we add the second and third row of $P-A$ to the first row of $P-A$, and then add the second and third column to the first column we see from (9) that

$$
\operatorname{det}\left[\begin{array}{ccc}
s & c_{2} & c_{3} \\
r_{2} & p_{22}-a_{22} & p_{23}-a_{23} \\
r_{3} & p_{32}-a_{32} & p_{33}-a_{33}
\end{array}\right]=0
$$

for every matrix $\left[\begin{array}{ll}p_{22} & p_{23} \\ p_{32} & p_{33}\end{array}\right]$ in $\Delta$, where

$$
s=3-\sum_{i, j=1}^{3} a_{i j}, \quad c_{k}=1-\sum_{i=1}^{3} a_{i k}, \quad r_{k}=1-\sum_{j=1}^{3} a_{k j}, \quad k=2,3 .
$$


Suppose that $s \neq 0$. Then (10) must hold for $c_{2}=c_{3}=0$, since any multiple of the first column can be added to any other column without changing the determinant. If $s \neq 0$ and $c_{2}=c_{3}=0$ in (10), we see that

$$
\begin{array}{r}
a_{22} a_{33}-\left(a_{23}-1\right)\left(a_{32}-1\right)=0, \\
a_{22} a_{33}-\left(a_{23}-1\right) a_{32}=0, \\
a_{22} a_{33}-a_{23}\left(a_{32}-1\right)=0, \\
\left(a_{22}-1\right) a_{33}-a_{23} a_{32}=0, \\
a_{22}\left(a_{33}-1\right)-a_{23} a_{32}=0 .
\end{array}
$$

It is not difficult to show that this system of equations has no solution. Hence, $s=0$.

Since $s=0$, from (10) we obtain a homogeneous system of five linear equations in the four unknowns $c_{2} r_{2}, c_{2} r_{3}, c_{3} r_{2}, c_{3} r_{3}$. Since the coefficient matrix for this system has rank four, we see that

$$
r_{2}=r_{3}=0 \text { or } c_{2}=c_{3}=0 .
$$

Therefore, since $s=0, A \varepsilon=\varepsilon$ or $A^{T} \varepsilon=\varepsilon$. Therefore, $\Gamma$ is an $(\varepsilon, \varepsilon)$-set. If any equation is removed from system (11), then the remaining system has a solution. Hence $\Gamma$ is a minimal $(\varepsilon, \varepsilon)$-set. It now follows from Lemma 3 that every set of five 3-square permutation matrices is a minimal $(\varepsilon, \varepsilon)$-set.

We have determined by machine calculation that the 12 matrices

$$
\begin{aligned}
& {\left[\begin{array}{llll}
1 & 0 & 0 & 0 \\
0 & 1 & 0 & 0 \\
0 & 0 & 1 & 0 \\
0 & 0 & 0 & 1
\end{array}\right],\left[\begin{array}{llll}
1 & 0 & 0 & 0 \\
0 & 0 & 1 & 0 \\
0 & 0 & 0 & 1 \\
0 & 1 & 0 & 0
\end{array}\right],\left[\begin{array}{llll}
0 & 1 & 0 & 0 \\
0 & 0 & 1 & 0 \\
0 & 0 & 0 & 1 \\
1 & 0 & 0 & 0
\end{array}\right],\left[\begin{array}{llll}
0 & 1 & 0 & 0 \\
0 & 0 & 0 & 1 \\
1 & 0 & 0 & 0 \\
0 & 0 & 1 & 0
\end{array}\right],} \\
& {\left[\begin{array}{llll}
0 & 1 & 0 & 0 \\
0 & 0 & 0 & 1 \\
0 & 0 & 1 & 0 \\
1 & 0 & 0 & 0
\end{array}\right],\left[\begin{array}{llll}
0 & 0 & 0 & 1 \\
0 & 0 & 1 & 0 \\
1 & 0 & 0 & 0 \\
0 & 1 & 0 & 0
\end{array}\right],\left[\begin{array}{llll}
0 & 0 & 1 & 0 \\
0 & 1 & 0 & 0 \\
1 & 0 & 0 & 0 \\
0 & 0 & 0 & 1
\end{array}\right],\left[\begin{array}{llll}
0 & 0 & 1 & 0 \\
0 & 0 & 0 & 1 \\
0 & 1 & 0 & 0 \\
1 & 0 & 0 & 0
\end{array}\right],} \\
& {\left[\begin{array}{llll}
0 & 0 & 0 & 1 \\
1 & 0 & 0 & 0 \\
0 & 1 & 0 & 0 \\
0 & 0 & 1 & 0
\end{array}\right],\left[\begin{array}{llll}
0 & 0 & 1 & 0 \\
0 & 1 & 0 & 0 \\
0 & 0 & 0 & 1 \\
1 & 0 & 0 & 0
\end{array}\right],\left[\begin{array}{llll}
0 & 1 & 0 & 0 \\
1 & 0 & 0 & 0 \\
0 & 0 & 1 & 0 \\
0 & 0 & 0 & 1
\end{array}\right],\left[\begin{array}{llll}
0 & 0 & 1 & 0 \\
1 & 0 & 0 & 0 \\
0 & 0 & 0 & 1 \\
0 & 1 & 0 & 0
\end{array}\right],}
\end{aligned}
$$

over $G F(2)$ form a minimal $(\varepsilon, \varepsilon)$-set. There also exist minimal $(\varepsilon, \varepsilon)$ sets of 4-square permutation matrices over $G F(2)$ of cardinality 13 , but there exist none of cardinality less than 12 . 


\section{REFERENCE}

1. R. A. Brualdi and H. W. Wielandt, A spectral characterization of stochastic matrices, Linear Algebra and Appl. 1 (1968), no. 1, 65-71. MR 36 \#6435.

Department of Mathematics, University of Alabama, Huntsville, Alabama 35807 (Current address of P. M. Gibson)

Current address (E. T. Beasley): Department of Computer Science, Ohio State University, Columbus, Ohio 43210 